\title{
Real-Time Killing Assays to Assess the Potency of a New Anti-Simian Immunodeficiency Virus Chimeric Antigen Receptor T Cell
}

\author{
Françoise Haeseleer, ${ }^{1,2}$ Karsten Eichholz, ${ }^{2}$ Semih U. Tareen, ${ }^{3, *}$ Nami Iwamoto, ${ }^{4}$ Mario Roederer, ${ }^{4}$ \\ Frank Kirchhoff, ${ }^{5}$ Haesun Park, ${ }^{6}$ Afam A. Okoye, ${ }^{6}$ and Lawrence Corey ${ }^{1,2,7}$
}

\begin{abstract}
The success of chimeric antigen receptor (CAR) T cell therapies for treating leukemia has resulted in a booming interest for the technology. Expression of a CAR in T cells allows redirection of their natural cytolytic activity toward cells presenting a specific designated surface antigen. Although CAR $\mathrm{T}$ cell therapies have thus far shown promising results mostly in B cell malignancy trials, interest in their potential to treat other diseases is on the rise, including using CAR T cells to control human immunodeficiency virus infection. The assessment of CAR T cell potency toward specific targets in vitro is a critical preclinical step. In this study, we describe novel assays that monitor the cytotoxicity of candidate CAR T cells toward simian immunodeficiency virus (SIV) infected CD4 T cells. The assays involve live cell imaging using a fluorescence microscopy system that records in real time the disappearance or appearance of targets infected with SIV carrying a fluorescent protein gene. The assays are highly reproducible, and their rapid turn around and reduced cost present a significant advance regarding the efficient preclinical evaluation of CAR $\mathrm{T}$ cell constructs and are broadly applicable to potential human diseases that could benefit from CAR T cell therapy.
\end{abstract}

Keywords: CAR T cell, cytotoxicity, immunodeficiency virus, fluorescent proteins, real-time

\section{Introduction}

$\mathbf{T}$ HE DEVELOPMENT OF cell therapies that utilize the administration of in vitro modified autologous cells has emerged as a robust technology for treating human diseases. Chimeric antigen receptor (CAR) expressing T cells are manipulated in vitro to express an antigen-binding peptide or antibody-based CAR that often targets a tumorspecific antigen. CAR T cells mediate cytotoxic activity against target cells through their intracellular signaling domains. ${ }^{1,2}$ CAR T cell therapies have been used predominantly to treat hematologic malignancies, but their use for other diseases, such as HIV/AIDS, is increasingly being explored. ${ }^{3-6}$
About 38 million people are chronically infected with human immunodeficiency virus (HIV) throughout the world, and only about $67 \%$ of these people are receiving antiretroviral therapy (ART). ${ }^{7}$ Although ART is successful in controlling viral load in the peripheral blood, it does not prevent HIV from establishing persistent viral reservoirs in diverse tissues. $^{8-10}$ Upon ART interruption, low levels of virus replication or reactivation of latently infected cells (i.e., reservoirs) results in viral rebound.

In an attempt to cure HIV, CAR T cell therapy has been investigated for specifically targeting remaining reservoirs of HIV infected cells in patients. Previous studies have investigated the therapeutic potential of such an approach using CAR T cells based on the extracellular domain of CD4 or

\footnotetext{
${ }^{1}$ Department of Laboratory Medicine, University of Washington, Seattle, Washington, USA.

${ }^{2}$ Vaccine and Infectious Disease Division, Fred Hutchinson Cancer Research Center, Seattle, Washington, USA.

${ }^{3}$ Juno Therapeutics, Seattle, Washington, USA.

${ }^{4}$ Vaccine Research Center, National Institute of Allergy and Infectious Diseases, National Institutes of Health, Bethesda, Maryland, USA.

${ }^{5}$ Institute of Molecular Virology, Ulm University Medical Center, Ulm, Germany.

${ }^{6}$ Vaccine and Gene Therapy Institute and Oregon National Primate Research Center, Oregon Health \& Science University, Beaverton, Oregon, USA.

${ }^{7}$ Department of Medicine, University of Washington, Seattle, Washington, USA.

*Current affiliation: Sana Biotechnology, Seattle, Washington, USA.
} 
broadly neutralizing antibodies recognizing a conserved epitope of HIV gp120 envelope. ${ }^{11,12}$ Methods to assess the ability of CAR T cells to kill HIV-infected cells in vitro are thus important for selecting the most efficient CAR T cells during preclinical development of antiviral therapies. In this study, using a simian model for HIV infection, we developed novel cytotoxic assays to evaluate the potency of CAR T cells against simian immunodeficiency virus (SIV)-infected CD4 T cells.

Multiple methods exist to assess the cytolytic activity of T cells. The chromium release assay has been the standard for assessing T cell cytolytic efficiency, but has the disadvantage of requiring radioactive isotopes. ${ }^{13}$ Indirect methods such as measurement of interferon gamma release by $\mathrm{T}$ cells assess the activation of effector $\mathrm{T}$ cells, but not target cell killing directly. ${ }^{14,15}$ Multiple assays that quantify the release of cell components from damaged target cells or use flow cytometric analysis have also been developed to assess cell-mediated cytotoxicity. ${ }^{16-22}$

Unfortunately, these assays have caveats such as requiring high cell viability or not providing data for multiple timepoints. In addition, many of these methods rely on cells expressing reporter genes and would be difficult to adapt for primary $\mathrm{T}$ cells. Impedance-based approaches are another type of assay that allows one to quantify the cytolytic activity of immune cells in real time but require adherent cells as targets or development of tethering approaches, when possible, with antibodies specific for suspension target cells. ${ }^{23}$

In this article, we describe killing assays that assess, in real-time, the cytotoxic activity of SIV envelope-specific CAR T cells against SIV-infected cells. These assays allow assessment of killing potency as frequently as needed and for as long as necessary after addition of effector cells to target cells. They can be easily adapted to monitor any CAR T cellmediated cytolysis of target cells infected with a recombinant virus encoding a fluorescent protein (FP).

\section{Materials and Methods}

\section{Generation of CAR T cells}

The CAR was generated with the antigen-binding domain of the ITS06.01 monoclonal antibody $(\mathrm{mAb})$ that targets the V1 epitope of SIV envelope. ${ }^{24}$ The single-chain variable fragment ( $\mathrm{scFv}$ ) of the ITS06.01 $\mathrm{mAb}$ is in a variable heavylight chain orientation fused through a medium size linker of 120 amino acids and was added to a CD28 transmembrane region, a $4-1 \mathrm{BB}$ intracellular costimulatory domain, and a CD3 $\xi$ signaling domain.

The ITS06 CAR was fused to a truncated epidermal growth factor receptor (EGFRt) as a marker to identify transduced cells using a Thosea asigna virus $2 \mathrm{~A}$ (T2A) self-cleavage peptide. This expression cassette was then cloned together with a woodchuck hepatitis virus post-transcriptional regulatory element (WPRE) for increased gene expression into a SIV-based lentiviral vector (a generous gift from Dr. Nienhuis, St June Children's Research Hospital, Memphis, TN). ${ }^{25}$ These elements were cloned between the AgeI and NotI site of the pCL20c MSCV-GFP (green fluorescent protein) plasmid downstream of the murine stem cell virus (MSCV) promoter using the NEBuilder HiFi DNA Assembly (New England Biolabs). For the control lentivirus, the EGFRt and WPRE were also cloned between the AgeI and Not I site of the pCL20c MSCV-GFP plasmid without the CAR element.
For the production of ITS06 CAR-EGFRt or EGFRt lentiviruses, Lenti- $X^{\mathrm{TM}} 293 \mathrm{~T}$ cells (Takara Bio) in Dulbecco's modified Eagle's medium containing $10 \%$ fetal bovine serum (FBS) and $100 \mathrm{U} / \mathrm{mL}$ Pen/Strep were transfected using the standard calcium phosphate method with four plasmids, $12 \mu \mathrm{g}$ of the CAR transfer vector, $6 \mu \mathrm{g}$ of the pCAG-SIVgprre plasmid carrying the gag/pol and rev responsive element, $4 \mu \mathrm{g}$ of the rev/tat expression plasmid pCAG4-RTR-SIV, and $3 \mu \mathrm{g}$ of the pMD2.CocalG containing the glycoprotein $\mathrm{G}$ of the cocal virus (a generous gift from Dr. Kiem, Fred Hutchinson Cancer Research Center, Seattle, WA). ${ }^{26}$

Sixteen hours after transfection, the HEK293T cells were washed with phosphate-buffered saline (PBS), and fresh media was added. The lentivirus-containing media was harvested 24 and $48 \mathrm{~h}$ later and cleared by centrifugation at 1,000 $g$ for $5 \mathrm{~min}$ followed by filtration on a $0.45 \mu \mathrm{m}$ Millipore filter. The lentivirus preparation was then concentrated by ultracentrifugation at 74,000 $\mathrm{g}$ for $2 \mathrm{~h}$ at $4^{\circ} \mathrm{C}$, resuspended in PBS ( $\sim 50 \times$ concentration) by incubation overnight at $4^{\circ} \mathrm{C}$, and then stored at $-80^{\circ} \mathrm{C}$.

The lentivirus preparation was then titrated by adding various amounts of lentivirus to Jurkat cells cultured in RPMI media supplemented with $10 \%$ FBS and $100 \mathrm{U} / \mathrm{mL}$ Pen/Strep on retronectin-coated plates with $4 \mu \mathrm{g} / \mathrm{mL}$ polybrene followed by spinoculation for $2 \mathrm{~h}$ at $1,200 \mathrm{~g}$. The percentage of transduced Jurkat cells was quantified by flow analysis for EGFRt using the anti-EGFRt cetuximab mAb (Erbitux, PE conjugated at Juno Therapeutics).

\section{Enrichment and transduction of CD4 and CD8 rhesus macaque $T$ lymphocytes}

Frozen peripheral blood mononuclear cells (PBMCs) from rhesus macaques (Macaca mulatta) of Indian genetic background were obtained from the Oregon National Primate Research Center in accordance with standards of the Center's Institutional Animal Care and Use Committee and the $\mathrm{Na}$ tional Institutes of Health Guide for the Care and Use of Laboratory Animals.

$\mathrm{CD}^{+}$and $\mathrm{CD}^{+} \mathrm{T}$ cells were enriched from PBMCs by immunomagnetic negative selection (EasySep nonhuman primate [NHP]; STEMCELL Technologies). $\mathrm{CD}^{+}$and $\mathrm{CD}^{+}$ T cells were cultured in X-VIVO 15 media (Lonza) supplemented with $10 \% \mathrm{FBS}, 100 \mathrm{U} / \mathrm{mL}$ Pen/Strep, $1 \times$ GlutaMAX, and $50 \mathrm{IU} / \mathrm{mL}$ human recombinant IL-2 (Peprotech). CD4 ${ }^{+}$ and $\mathrm{CD}^{+} \mathrm{T}$ cell expansion was initiated by the addition of ImmunoCult NHP CD2/CD3/CD28 T cell activator (STEMCELL Technologies) and incubation for $72 \mathrm{~h}$ at $37^{\circ} \mathrm{C}$ in a humidified $5 \% \mathrm{CO}_{2}$ atmosphere.

$\mathrm{CD}^{+}$and $\mathrm{CD}^{+} \mathrm{T}$ cells mixed at a ratio of about 1:1 were plated on RetroNectin (Takara Bio)-coated plates and transduced by adding CAR lentivirus at a multiplicity of infection (MOI) of $\sim 2$ with $4 \mu \mathrm{g} / \mathrm{mL}$ polybrene followed by spinoculation for $2 \mathrm{~h}$ at 1,200 $\mathrm{g}$. Cells were washed about $24 \mathrm{~h}$ after transduction and expanded in fresh complete X-VIVO 15 media.

Four days after transduction, $\mathrm{T}$ cells were analyzed for EGFRt expression by flow cytometry using PE-anti-EGFRt, BV421 anti-CD4 (OKT4; BioLegend), and APC-Cyanine7 anti-CD8 (SK1; BioLegend). EGFRt expression was analyzed using FlowJo and sequential gating on lymphocytes, single cells, and then CD4 or CD8 T cells. 
Analysis of gp140 protein binding to CD8 CAR T cells was performed by incubation of CD8 CAR T cells or CD8 EGFR $\mathrm{T}$ cells with $10 \mu \mathrm{g} / \mathrm{mL}$ of SIVmac239 gp140 (Immune Technology Corp.) in PBS $+1 \%$ bovine serum albumin (BSA) at room temperature for $30 \mathrm{~min}$. For the detection of cellbound gp140, SIV-ENV specific ITS52 $\mathrm{mAb}^{24}$ biotinylated with EZ-Link Sulfo-NHS-LC-Biotin (Thermo Scientific) following the manufacturer's instructions was added to the washed cells and incubated for $30 \mathrm{~min}$ at room temperature followed by a 15-min incubation with BV421 streptavidin (BioLegend) and analyzed by flow cytometry.

\section{Production of SIV-GFP and SIV-mruby3 viruses and CD4 $T$ cell infection}

To generate the SIVmac239 viruses that express FPs, an expression cassette containing an internal ribosome entry site (IRES) element followed by the cDNA encoding the enhanced green fluorescent protein (EGFP) or a monomeric red fluorescent protein (mRuby3) was introduced downstream of the nef gene into a plasmid containing the full-length SIV $_{\text {MAC239 }}$ proviral DNA. For virus production, HEK293T cells were transfected with $20 \mu \mathrm{g}$ of the SIVmac239-EGFP or SIVmac239mruby3 plasmid DNA using the calcium phosphate method.

Sixteen hours after transfection, the HEK293T cells were washed with PBS, and fresh media was added. The SIVmac239containing supernatant was collected $48 \mathrm{~h}$ later, cleared by centrifugation for $5 \mathrm{~min}$ at $1,000 \mathrm{~g}$, and filtered on a $0.45 \mu \mathrm{m}$ Millipore filter. The EGFP or mruby3-SIVmac239 viruses simian immunodeficiency virus-fluorescent protein (SIVFP) were then concentrated by mixing one volume of Lenti$\mathrm{X}$ concentrator (Takara Bio) with three volumes of cleared supernatant. After an incubation of $30 \mathrm{~min}$ at $4^{\circ} \mathrm{C}$, the SIV-FP were pelleted by centrifugation for $45 \mathrm{~min}$ at $1,500 \mathrm{~g}$, resuspended in PBS (final concentration $\sim 25 \times$ ), and aliquots were stored at $-80^{\circ} \mathrm{C}$. Stock of SIV-FP viruses was titrated using TZM-bl cells as described by Wei et al. ${ }^{27}$

After expansion for 8-10 days of CD4 T cells prepared as described above, infection was performed by adding $20 \mu \mathrm{L}$ of concentrated SIV-FP viruses to $10^{5} \mathrm{CD} 4$ cells ( $\sim$ MOI 0.5 ) plated on RetroNectin-coated 96-well plates followed by spinoculation for $2 \mathrm{~h}$ at $1,200 \mathrm{~g}$. Cells were then incubated for at least 3 days at $37^{\circ} \mathrm{C}$ for the virus propagation and for the accumulation of FPs. The percentage of SIV-FP-infected cells was assessed using flow cytometry after gating on lymphocytes and single cells.

\section{Real-time killing assay}

Fluorescent SIV-FP-infected targets at 20,000 cells per $50 \mu \mathrm{L}$ of X-VIVO 15 media were plated on BioCoat poly-Dlysine coated flat bottom 96-well plates (Corning). The assay was performed with preparation of target CD4 T cells containing $\sim 20 \%$ of SIV infected cells. After a $2 \mathrm{~h}$ incubation at $37^{\circ} \mathrm{C}$, effector CAR $\mathrm{T}$ cells or effector EGFR $\mathrm{T}$ cells in $200 \mu \mathrm{L}$ complete $\mathrm{X}$-VIVO 15 media were added in triplicate at various effector-to-target $(\mathrm{E}: \mathrm{T})$ ratios as indicated; the number of effector CAR T cells being calculated based on the percentage of transduced EGFRt-positive cells. Plates were incubated at $37^{\circ} \mathrm{C}$ for up to 3 days, and five images were recorded per well every $3 \mathrm{~h}$ and analyzed with the IncuCyte image analysis software.
The killing potency of the anti-SIV CAR T cells was assessed by comparing the number of fluorescent targets over time relative to their number at time 0 . To evaluate the viral spread to EGFR $\mathrm{T}$ cells during the killing assay, SIV-FP infected CD4 T cells were cocultured with EGFR T cells at the indicated E:T ratios for 3 days as described above and in triplicate. The percentage of infected $\mathrm{EGFR}^{+}$and $\mathrm{EGFR}^{-}$ $\mathrm{CD}^{-} \mathrm{T}$ cells was determined using flow cytometry after gating for lymphocytes, single cells, and $\mathrm{CD} 8^{-}$. $\mathrm{CD} 8^{-}$was used as a surrogate gate for CD4 T cells as SIV-infected cells do not express CD4 or express it at a low level.

\section{Assessment of CAR T cell potency by real-time monitoring of cell infection}

For the infection of CD4 rhesus macaque cells, a master mix of CD4 $\mathrm{T}$ cells/SIV-FP virus was prepared by adding $4 \mu \mathrm{L}$ of concentrated viruses per 20,000 CD 4 T cells ( $\sim$ MOI 0.5 ) in $50 \mu \mathrm{L} \mathrm{X-VIVO} \mathrm{media.} \mathrm{The} \mathrm{target/SIV} \mathrm{mix} \mathrm{was} \mathrm{plated}$ on BioCoat poly-D-lysine coated flat bottom 96-well plate followed by spinoculation for $2 \mathrm{~h}$ at $1,200 \mathrm{~g}$. The assay was then carried out similarly to the real-time killing assay as described above by adding effector $\mathrm{T}$ cells at various $\mathrm{E}: \mathrm{T}$ ratios in triplicate and recording five images per well every $3 \mathrm{~h}$. To evaluate the EGFR T cell infection over the time of the assay, 3 wells of a 96-well plate were set up in the same condition; that is, target cells were infected with SIV-FP and EGFR T cells were then added at various E:T ratios. After 3 days of coculture, the percentage of infected $\mathrm{EGFR}^{+}$and $\mathrm{EGFR}^{-} \mathrm{CD}^{-} \mathrm{T}$ was determined using flow cytometry after gating for lymphocytes, single cells, and $\mathrm{CD}^{-}$.

\section{Flow cytometry}

Jurkat cells or rhesus macaque T cells were washed with PBS containing $1 \%$ BSA and incubated with the indicated antibodies for $30 \mathrm{~min}$ at room temperature. Samples were then washed with PBS-BSA and fixed in $2 \%$ paraformaldehyde in PBS. Flow cytometry was performed on a BD LSRII flow cytometer, and the data were analyzed using the FlowJo 10 software.

\section{Statistical analysis}

Data are presented as the mean \pm standard error of the mean. Statistical significance was analyzed by Student's $t$-test for each timepoint.

\section{Results}

\section{Preparation of effector cells for killing assay}

Our goal was to develop an assay that will allow rapid screening of antiviral CAR T cells for killing potency. First, we generated a lentiviral vector that encodes an anti-SIV CAR based on the scFv of the ITS06.01 mAb. This mAb was selected based on its high binding affinity for gp140 of various SIV strains ${ }^{24}$ and neutralization of both tier 1 and 2 isolates of SIVsmE660 and SIVmac251, but not the tier 3 SIVmac239. The anti-CAR was also composed of a 4-1BB intracellular costimulatory domain and a $\mathrm{CD} 3 \xi$ activation domain (Fig. 1A). ${ }^{24}$ To easily assess the lentiviral transduction efficiency into T cells, the DNA construct also included a truncated version of the EGFR that lacks the EGF binding domain and 


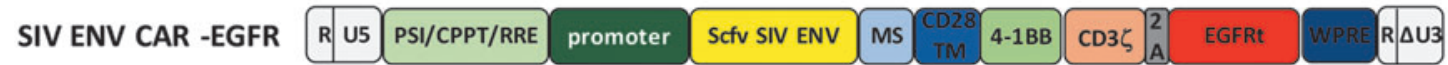

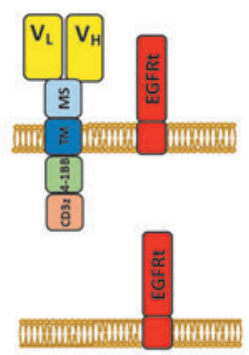

EGFR R U U PSI/CPPT/RRE promoter EGFRt WPRE R $\triangle \mathrm{UU}$

B
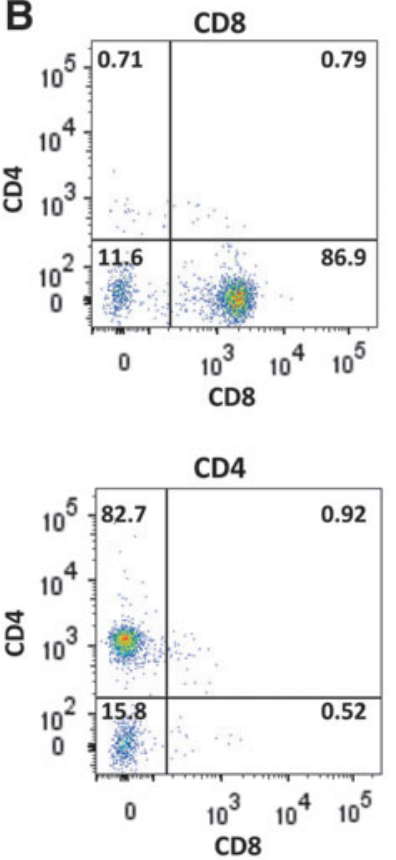
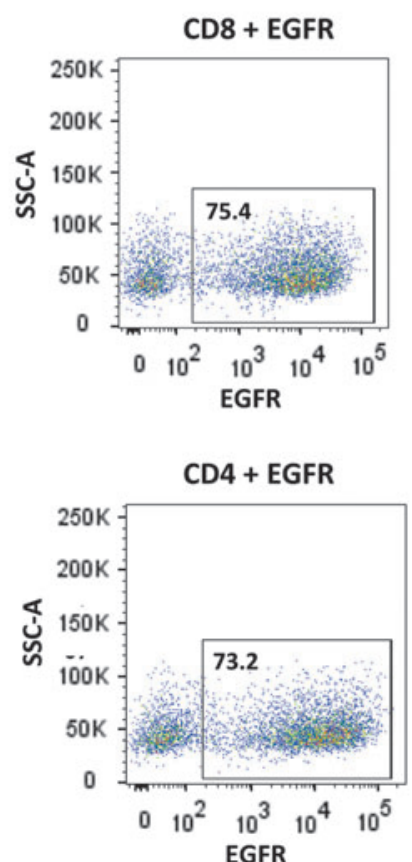
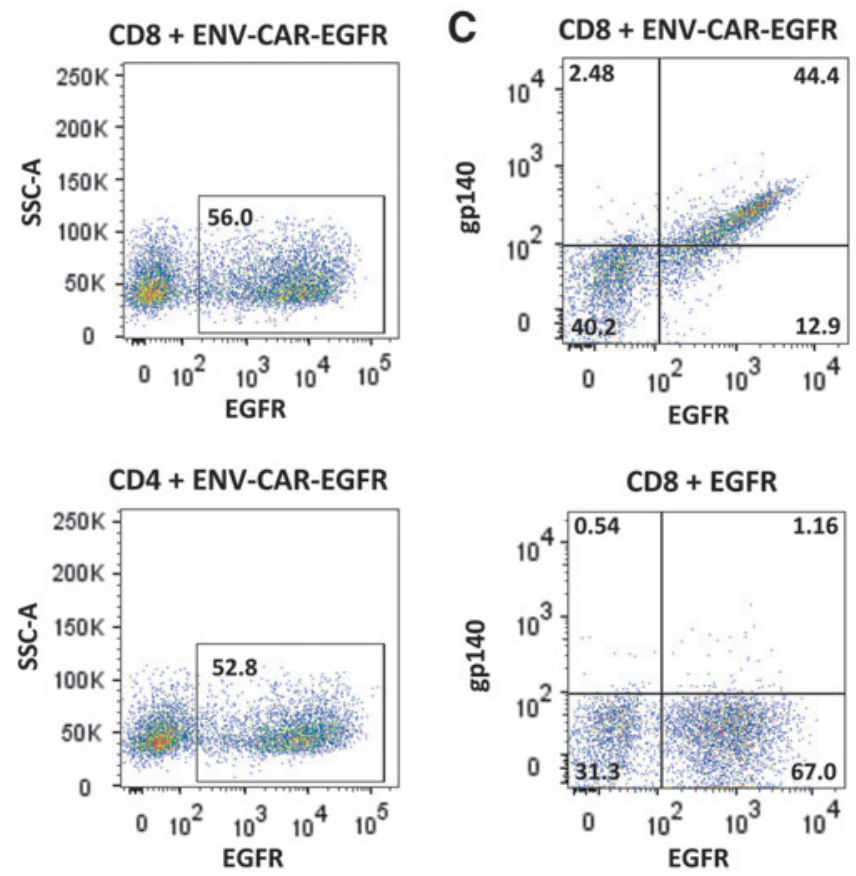

FIG. 1. Assembly of viral vectors and preparation of effectors for the killing assays. (A) Schematic diagram of the antiSIV ENV CAR and the control EGFR lentiviral vectors (left) and of the expressed proteins at the cell surface (right). (B) Flow cytometry analysis of CAR T cells for the expression of EGFRt in CD8 (top) and CD4 (bottom) T cells transduced with the SIV ENV CAR or EGFR lentiviral vectors shown in (A). Assessment of the CD8 and CD4 T cell preparations (left) and of EGFRt expression in cells transduced with the EGFR lentivirus (middle) or SIV ENV CAR lentivirus (right). (C) Env binding to CD8 CAR T cells. CD8 CAR T cells or CD8 EGFR T cells were incubated with SIVmac239 gp140 proteins followed by incubation with biotinylated anti-Env mAb and BV421-streptavidin. CAR, chimeric antigen receptor; EGFR, epidermal growth factor receptor; mAb, monoclonal antibody; SIV, simian immunodeficiency virus.

intracellular domains. This truncated protein can be detected on the cell surface using an anti-EGFR mAb (cetuximab).

Rhesus macaques have been used extensively as animal models for HIV infection. To achieve a high level of simian T cell transduction, CAR-EGFRt DNA was cloned into a SIVbased lentiviral system composed of four plasmids that provides the necessary viral functions for lentiviral DNA packaging. ${ }^{25}$ The helper plasmid encoding the vesicular stomatitis virus $\mathrm{G}$ protein was replaced with a plasmid encoding the cocal virus envelope glycoprotein, which has been shown to increase the transduction efficiency of lentiviral vectors in macaque cells. ${ }^{26}$

CD4 and CD8 T cells were enriched from rhesus macaque PBMCs by negative selection using a commercially available kit. After expansion for 3 days, CD4 and CD8 T cells were mixed at a ratio of about 1:1, similarly to the optimized CAR $\mathrm{T}$ cell infusion products used in some clinical trials, ${ }^{28-30}$ and transduced with lentivirus carrying the SIV-CAR. The per- centage of transduced cells was then assessed by analyzing the expression of EGFRt by flow cytometry. On average, a transduction efficiency of 50\%-80\% was observed using this lentiviral system (Fig. 1B).

To verify that the CAR scFv binding domain has the ability to bind target antigen, binding of SIVmac239 gp140 to CAR $\mathrm{T}$ cells was analyzed by flow cytometry using CD8 CAR T cells. CD4 CAR T cells could not be used for this analysis because of the intrinsic binding affinity of gp140 to the CD4 receptor. Binding of SIV gp140 was detected to EGFRt ${ }^{+}$ CAR T cells (Fig. 1C). We did not detect binding of gp140 to cells expressing EGFRt only.

\section{Development of fluorescent target cells}

The development of a novel real-time imaging killing assay required the availability of fluorescent target cells. The susceptibility of NHP CD4 T cells to SIV infection is often 
low and highly variable among animals. We observed that CD4 T cell susceptibility to SIV infection was affected by several experimental parameters, including expansion of CD4 T cells for 8-10 days before infection, concentration of SIV, infection on RetroNectin-coated plates, and spinoculation. We observed a CD4 $\mathrm{T}$ cell rate of infection by SIV ranging from $\sim 1 \%$ to $25 \%$ (data not shown).

Because of the low rate of SIV infection, labeling the entire CD4 $\mathrm{T}$ cell population with a fluorescent cell tracking dye would result in a high background coming from the abundant noninfected CD4 cells. To overcome this issue, we developed two new recombinant SIVmac239 viruses encoding FPs by cloning the cDNA for the EGFP or mRuby3 FP in fusion with an IRES downstream of the nef gene (Fig. 2A). These SIV-FP viruses were infectious and replication competent and led to high levels of FP expression in up to $25 \%$ of CD4 infected cells (Fig. 2B). As previously reported for wild-type SIV, ${ }^{31}$ these SIV-FP viruses downregulate the expression of CD4 antigens on the surface of infected cells (Fig. 2B). Using these new SIV-FP recombinant viruses, we were able not only to eliminate the background signal but also to measure more accurately the CAR $\mathrm{T}$ cell cytotoxic activity against SIV-infected CD4 target cells (see Fig. 3).

\section{IncuCyte assay to assess real-time killing} of SIV-infected cells by anti-SIV gp120 CAR T cells

To evaluate the killing potency of anti-SIV CAR T cells toward SIV-infected CD4 $\mathrm{T}$ cells, we first determined the optimal number of SIV-FP-infected CD4 target cells to seed in 96-well plates, considering the variable percentage of infected cells obtained among the CD4 target cell preparations. The optimal amount of target cells to use for accurate quantification was 20,000 cells per well, which was also selected to avoid high cell density after addition of effector cells. CD4 T cells were infected with fluorescent SIV for a minimum of 3 days before the assay to reach a suitable percentage of infected targets (Fig. 3A).

On the day of the assay, target cells settled for at least $2 \mathrm{~h}$ after seeding in triplicate in 96-well plates. CAR T cells or EGFR control $\mathrm{T}$ cells were then added to the plate at various E:T ratios, and five images per well were recorded every $3 \mathrm{~h}$ for 3 days.

The killing potency of the CAR $\mathrm{T}$ cells toward SIVinfected cells was assessed by comparing the number of fluorescent target cells at each timepoint to their number at time 0 . Killing of SIV-infected cells was readily detectable at the first 3-h timepoint and increased over time as shown by the decrease in the percentage of fluorescent CD4 in the presence of the CAR T cells (blue curve, Fig. 3B). Killing was proportional to the ratio of effector cells and occurred between E:T ratios of 5:1 and 1:3 (Fig. 3B-D). As shown in Figure $3 \mathrm{~B}$ and D, cytotoxicity of control EGFR T cells toward infected targets was undetectable. The observed increase in the number of fluorescent cells with control $\mathrm{T}$ cells (red curve) is likely due to proliferation of infected targets and cell-to-cell viral spread. Noninfected CD4 cells were not killed by CAR T cells or EGFR T cells (Fig. 3E).

We investigated the possibility that $\mathrm{CD}^{+}{ }^{+} \mathrm{CAR} \mathrm{T}$ cells could become infected with SIV and subsequently affect analysis of the data using EGFR T cells as effectors because SIV-infected ITS06 CAR T cells could be killed before the expression of GFP. SIV-infected CD4 T cells and EGFR T cells were cocultured under the same conditions for the maximum assay time and then assessed for infection using flow cytometry. We gated on $\mathrm{CD}^{-} \mathrm{T}$ cells for $\mathrm{CD} 4$ targets as SIV-infected cells express no or low level of CD4. The number of $\mathrm{EGFR}^{+} \mathrm{CD}^{-} \mathrm{T}$ cells that became infected over time was negligible and did not affect assay quality (Fig. 3F). All together, these data indicate that this assay can be used for fast evaluation of the cytotoxicity of antiviral CAR T cells toward infected targets in real time.

A

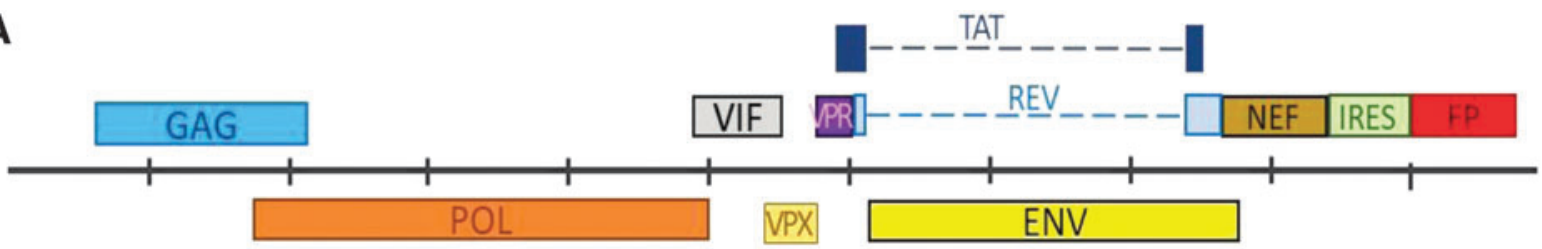

B

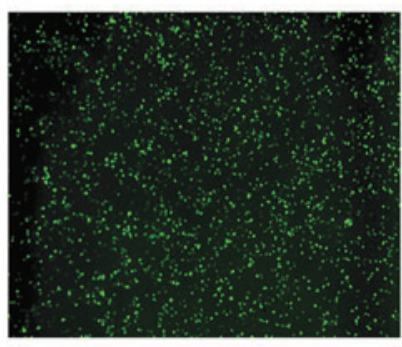

CD4 + SIV-GFP

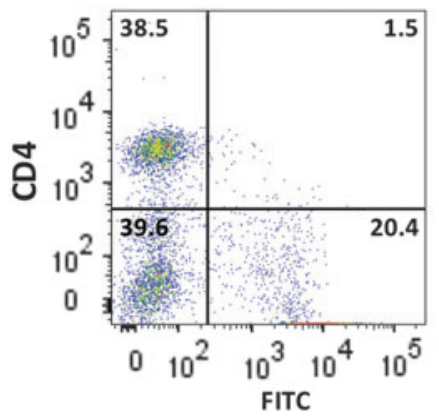

CD4 + SIV-ruby

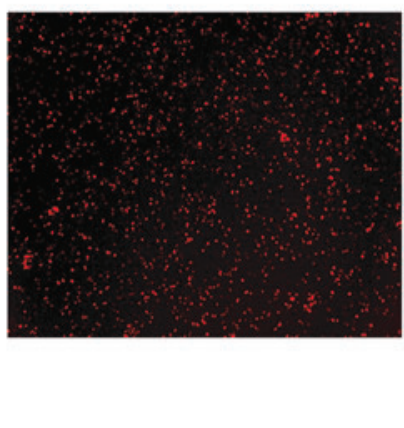

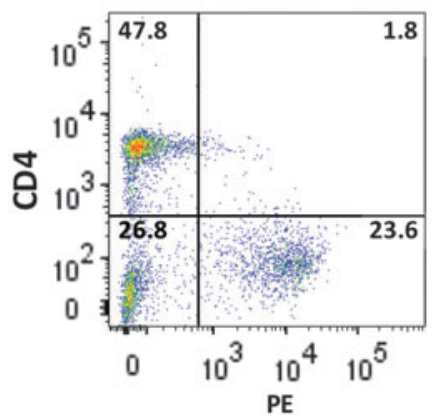

FIG. 2. Generation of fluorescent targets. (A) Scheme of the SIVmac239 genome with the IRES-FP cassette inserted downstream of the nef gene. (B) IncuCyte image and flow cytometry analysis of EGFP or mRuby 3 expression in purified CD4 T cells infected with the SIV-EGFP (left) or SIV-mRuby3 (right) virus. EGFP, enhanced green fluorescent protein; FP, fluorescent protein; IRES, internal ribosome entry site. 


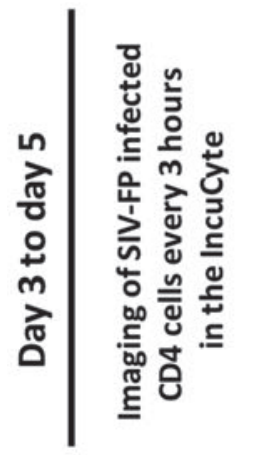

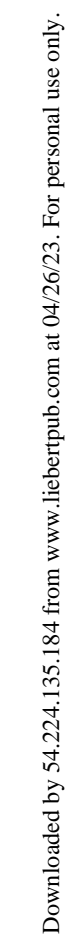
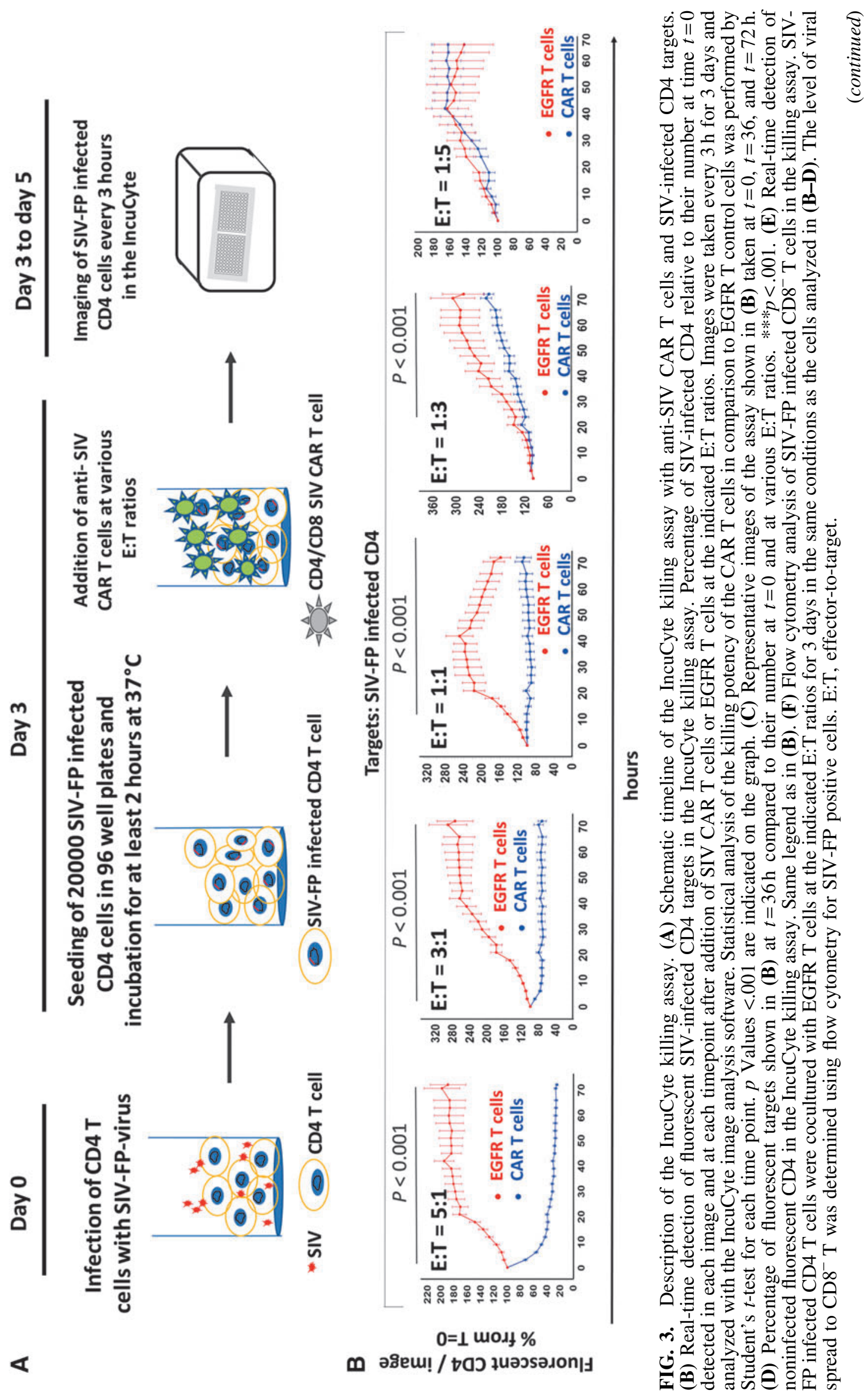


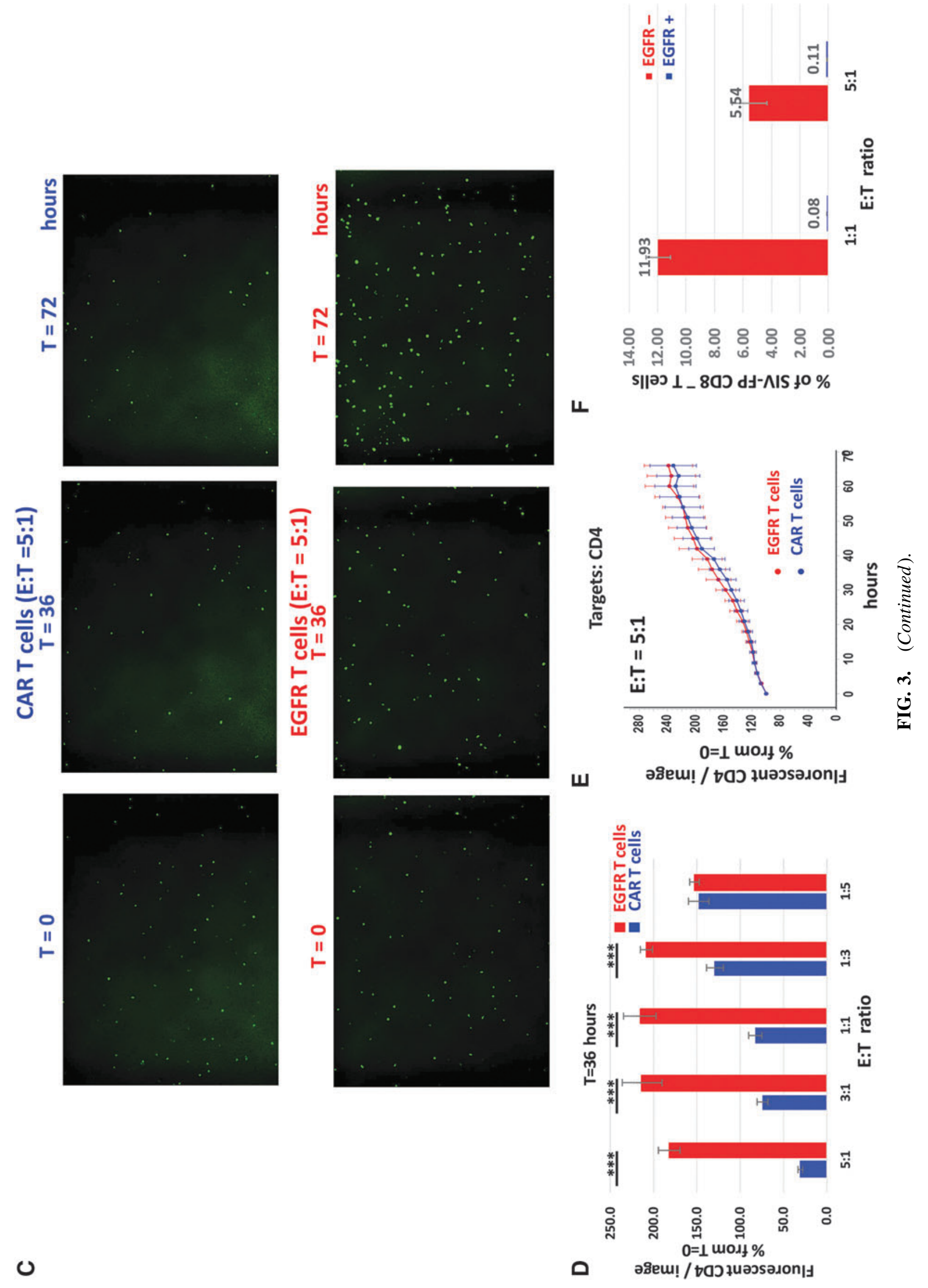



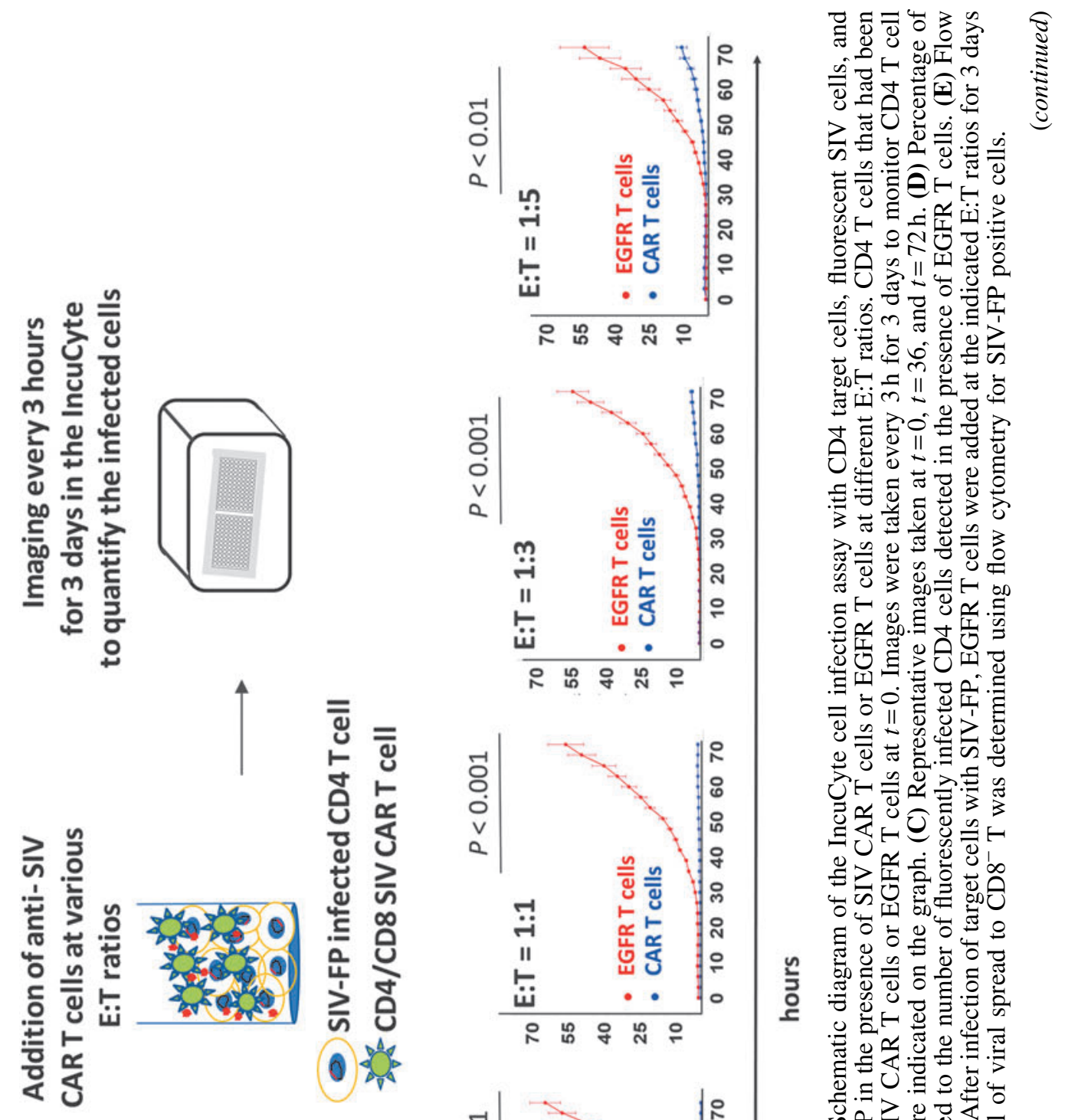

过

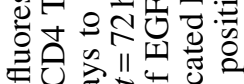

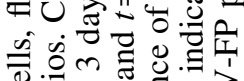

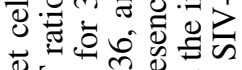

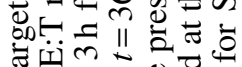

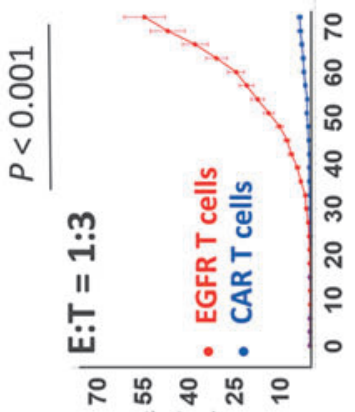

$\exists$ Iim

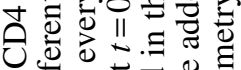

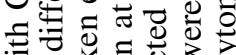

उ

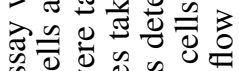

के ठ

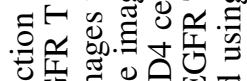

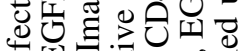

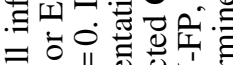

$\overline{8} 0$ 잉

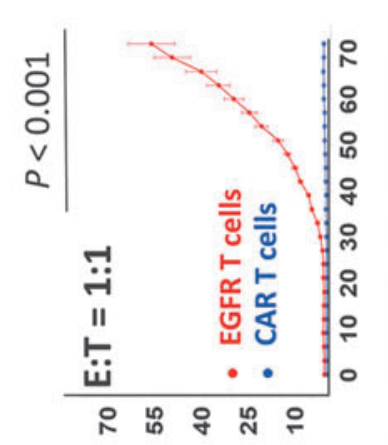

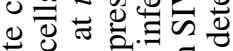

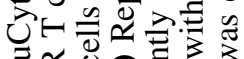

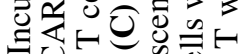

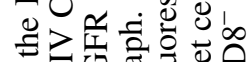

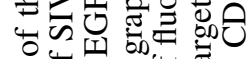

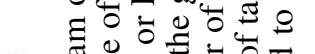

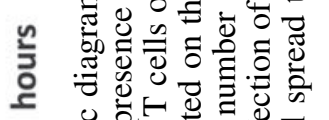
.气 巳.

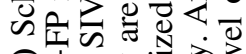

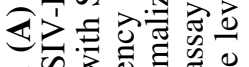

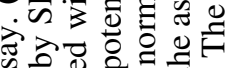

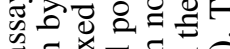

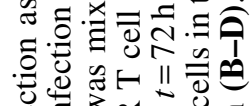

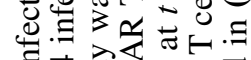
洁芯而事

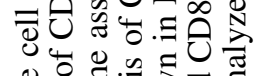
0ั0 Ð

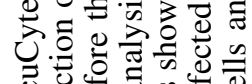

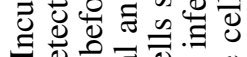

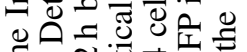

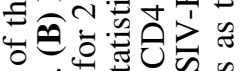

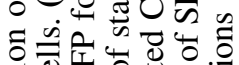

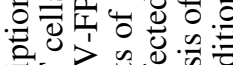

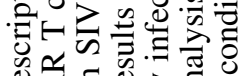

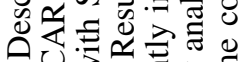

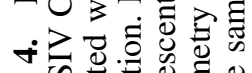
+.) 


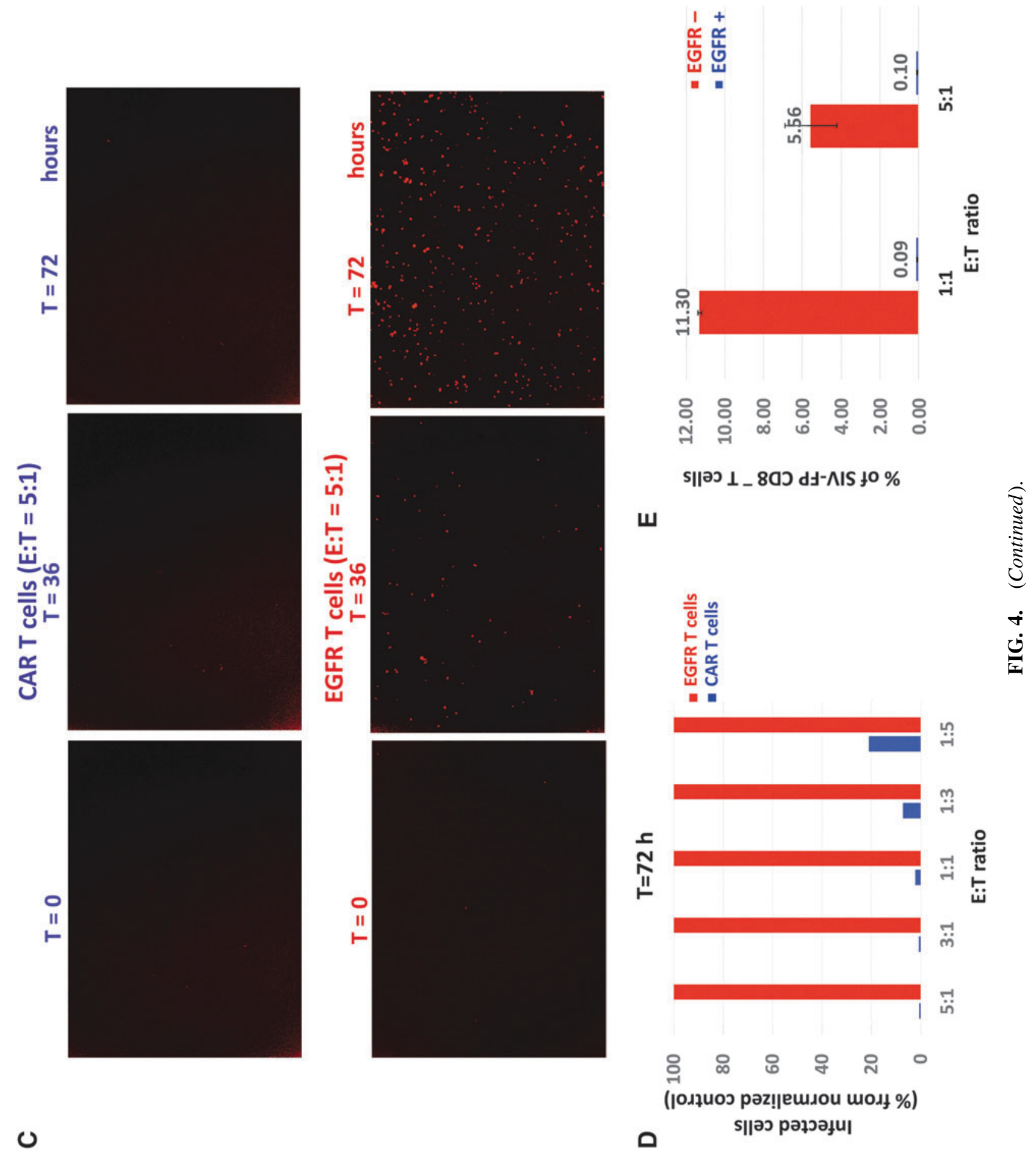




\section{Assessment of CAR T cell potency in killing SIV-infected cells upon infection}

Recently infected cells can contribute to the observed increase in viral load following ART interruption. A second assay was developed to evaluate the CAR $\mathrm{T}$ cell potency against freshly infected target cells. If CAR T cells kill CD4 T cells upon infection or SIV binding, expression of FPs would not occur.

For this second assay, SIV-FP was added first to CD4 T cells for $2 \mathrm{~h}$, as required for infection of the cells by spinoculation (Fig. 4A). Anti-SIV CAR T cells were then added to the 96-well plate at different E:T ratios, and cell infection was monitored by recording images every $3 \mathrm{~h}$ for 3 days. Three days after infection, fluorescently infected CD4 cells were barely detectable in the presence of CAR T cells between E:T ratios of 5:1 and 1:1 compared with EGFR T cells; and the number of infected cells remained small at the lowest E:T ratio of 1:5 (Fig. 4B-D). This effect of anti-SIV CAR T cells toward CD4 cells during infection is detected as soon as EGFP or mruby3 expression is visible starting $\sim 24 \mathrm{~h}$ after exposure to SIV-FP (Fig. 4B-D).

We also evaluated the percentage of CD4 CAR T cells that could become infected in this assay using EGFR $\mathrm{T}$ cells. After infection of the targets with SIV-FP, they were cocultured with EGFR T cells in the same condition and for the maximum time of the assay. Cells were collected from three wells and analyzed for infection using flow cytometry. The number of $\mathrm{EGFR}^{+} \mathrm{CD}^{-} \mathrm{T}$ cells that became infected over time was also negligible (Fig. 3F). Overall, these data demonstrate that this assay can assess the potency of CAR T cells in affecting cell infection.

\section{Discussion}

In this study, we developed new fast assays to evaluate the in vitro potency of antiviral CAR T cells in killing SIV targets. The assay described in Figure 3 analyzes directly in real time the killing of SIV-infected CD4 targets by anti-SIV CAR T cells. The assay described in Figure 4 assesses indirectly the ability of CAR T cells to affect cell infection and suppress target cells, which might occur through multiple pathways. In addition to CAR T cell killing of infected cells, SIV targets might be eliminated before viral entry when coated with virus particles. Activated CAR T cells might also secrete soluble factors that could inhibit SIV entry into CD4 T cells. ${ }^{32}$ This second assay is thus also valuable for testing additional damaging effects of the CAR T cells toward SIV target cells.

Various assays have been developed that assess the release of cell components from damaged or dead cells such as radioactive chromium, enzymes, and cytokines after exposure to activated $\mathrm{T}$ cells ${ }^{13,16-19}$ or that measure targets labeled with fluorescent dyes. ${ }^{20-22}$ Indirect methods such as measurement of interferon gamma or granzyme $\mathrm{B}$ release by $\mathrm{T}$ cells or intracellular granzyme and perforin upregulation in $\mathrm{T}$ cells assess the activation of effector $\mathrm{T}$ cells but not target cell killing. ${ }^{14,15,33,34}$ The evaluation of caspase 3 or granzyme B activity in target cells are other approaches to detect injury by effector $\mathrm{T}$ cells but do not demonstrate a direct result of the elimination of targets. ${ }^{35}$ Flow cytometry with anti-p24 antibodies can be used to quantify infected cells. ${ }^{36}$ Nevertheless, all these assays have similar drawbacks: for example, high background due to nonspecific labeling or release of assessed components from unhealthy cells and/or single time point data.
The assays described here have several advantages such as being fast, simple, and inexpensive. It is highly reproducible with no cell processing required after the addition of the target and effector cells to the plate and no need for commercial reagents. The analysis of the images is straightforward and rapid using IncuCyte software. In addition, the killing assay specifically monitors the killing of SIV-infected cells by CAR $\mathrm{T}$ cells in real time, allowing adaptation of image acquisition frequency and assay length as needed. While our assays typically lasted 2-3 days, CAR T cell potency could be detected as early as a few hours after addition of effector CAR T cells to SIV-infected targets. With a practically unlimited number of measurements, this assay also allows us to compare the kinetics of killing. Impedancebased assays are another option to quantify the cytolytic activity of immune cells in real time but require target cells to be attached to the electrodes and are thus less suitable to assess killing of nonadherent target cells such as primary CD4 T cells. ${ }^{23}$

The preparation of target cells is a critical factor in these assays. The low rate of infectivity of CD4 T cells by SIV in our simian model requires the use of viruses carrying a FP for the identification of the infected cells. Still, for other experimental models, target cells could be labeled with commercially available fluorescent dyes. We observed toxicity when labeling the primary rhesus macaque $\mathrm{T}$ cells with commercially available dyes reported as nontoxic for live cells and recommend instead the use of recombinant FP viruses for the identification of the infected cells when possible. This assay was usually performed with preparation of target cells containing $\sim 20 \%$ of infected cells. However, the assay could be performed with CD4 target preparations containing as little as $3 \%$ infected cells when $\mathrm{T}$ cells from some rhesus macaque animals were more refractory to infection by SIV.

The CAR T cells analyzed using these assays do not require protection because rhesus macaque CD4 $\mathrm{T}$ cells are poorly permissive to SIV infection when not conditioned (as described in our protocol for target preparation), and the timeframe of the assay is short. Negligible numbers of CAR T cells did get infected during the assay as evaluated with control EGFR CD4 T cells (Figs. 3F and 4E). However, for long-term in vivo studies or in vitro studies with highly permissive targets, CAR T cells should be protected against viral infection; for example, by disruption of the CCR5 gene in CAR T cells ${ }^{37}$ or by coexpression of gp 41 -derived peptides. ${ }^{38}$

Although the assay can be performed with any cell culture plates, we recommend the use of poly-lysine plates that promote adherence of the target cells and, thus, limit movement of nonadherent target cells at the well edges. However, the IncuCyte S3 instrument offers the option of recording images from the whole well that can compensate for this edge effect if it occurs.

\section{Conclusions}

Given the growing interest in developing novel cell therapies, assessing CAR T cell potency in vitro is an important step in preclinical development. We have demonstrated using the described methods that the ITS06.01-based CAR T cells kill SIV-infected CD4 T cells and affect the level of infection of target cells by SIV. Using both assays will ensure evaluation of cytolytic and noncytolytic effects of CAR T cells on 
SIV-infected cells. These methods are fast, cheap, and highly reliable. They can not only be used for large-scale screening of CAR T cells but also for quality control during CAR T cell manufacturing for immunotherapy. These assays were developed using anti-SIV CAR T cells and SIV-infected CD4, but can be adapted to any antiviral CAR T cells for which a virus with a FP marker is available.

\section{Authors' Contributions}

F.H. and L.C. conceived and designed the experiments. F.H. performed the experiments. K.E., S.U.T, N.I., M.R., F.K., H.P., and A.A.O. contributed reagents/materials/ advice. F.H and L.C. wrote the article. All authors read and commented on the article.

\section{Acknowledgments}

The authors thank Dr. Arthur Nienhuis (St June Children's Research Hospital, Memphis, TN) for kindly providing the SIV-based lentiviral vectors, Dr. Hans Peter Kiem (Fred Hutchinson Cancer Research Center, Seattle, WA) for the pMD2.Cocal.G expression plasmid, and Drs. Serge Barcy and Mindy Miner for suggestions and critiques on the article.

\section{Author Disclosure Statement}

No competing financial interests exist.

\section{Funding Information}

This work was supported by National Institute of Health grant UM1 A126623, the Deutsche Forschungsgemeinschaft (DFG), and by the Gilead Sciences HIV Cure Grant Program.

\section{References}

1. Gill S, June $\mathrm{CH}$ : Going viral: Chimeric antigen receptor T-cell therapy for hematological malignancies. Immunol Rev 2015;263:68-89.

2. Jensen MC, Riddell SR: Design and implementation of adoptive therapy with chimeric antigen receptor-modified $\mathrm{T}$ cells. Immunol Rev 2014;257:127-144.

3. Gill S, Maus MV, Porter DL: Chimeric antigen receptor T cell therapy: 25years in the making. Blood Rev 2016;30: 157-167.

4. Maus MV, Grupp SA, Porter DL, June CH: Antibodymodified T cells: CARs take the front seat for hematologic malignancies. Blood 2014;123:2625-2635.

5. Kim GB, Hege K, Riley JL: CAR Talk: How cancerspecific CAR T cells can instruct how to build CAR T cells to cure HIV. Front Immunol 2019;10:2310.

6. Haran KP, Hajduczki A, Pampusch MS, et al.: Simian immunodeficiency virus (SIV)-specific chimeric antigen receptor- $\mathrm{T}$ cells engineered to target $\mathrm{B}$ cell follicles and suppress SIV replication. Front Immunol 2018;9:492.

7. HIV/AIDS: Data and statistics. Available at https://www .who.int/hiv/data/en (2020), accessed August 12, 2020.

8. Chun TW, Stuyver L, Mizell SB, et al:: Presence of an inducible HIV-1 latent reservoir during highly active antiretroviral therapy. Proc Natl Acad Sci U S A 1997;94: 13193-13197.

9. Siliciano JD, Kajdas J, Finzi D, et al.: Long-term follow-up studies confirm the stability of the latent reservoir for HIV1 in resting CD4+ T cells. Nat Med 2003;9:727-728.
10. Estes JD, Kityo C, Ssali F, et al.: Defining total-body AIDS-virus burden with implications for curative strategies. Nat Med 2017;23:1271-1276.

11. Ghanem MH, Bolivar-Wagers S, Dey B, et al:: Bispecific chimeric antigen receptors targeting the CD4 binding site and high-mannose Glycans of gp120 optimized for antihuman immunodeficiency virus potency and breadth with minimal immunogenicity. Cytotherapy 2018;20:407-419.

12. Ali A, Kitchen SG, Chen ISY, Ng HL, Zack JA, Yang OO: HIV-1-Specific chimeric antigen receptors based on broadly neutralizing antibodies. J Virol 2016;90:69997006.

13. Brunner KT, Mauel J, Cerottini JC, Chapuis B: Quantitative assay of the lytic action of immune lymphoid cells on 51-Cr-labelled allogeneic target cells in vitro; inhibition by isoantibody and by drugs. Immunology 1968;14: 181-196.

14. Lalvani A, Pareek M: Interferon gamma release assays: Principles and practice. Enferm Infecc Microbiol Clin 2010;28:245-252.

15. Michel $\mathrm{N}$, Ohlschlager $\mathrm{P}$, Osen $\mathrm{W}$, et al.: $\mathrm{T}$ cell response to human papillomavirus $16 \mathrm{E} 7$ in mice: Comparison of $\mathrm{Cr}$ release assay, intracellular IFN-gamma production, ELISPOT and tetramer staining. Intervirology 2002;45:290-299.

16. Corey MJ, Kinders RJ, Brown LG, Vessella RL: A very sensitive coupled luminescent assay for cytotoxicity and complement-mediated lysis. J Immunol Methods 1997;207: 43-51.

17. Korzeniewski C, Callewaert DM: An enzyme-release assay for natural cytotoxicity. J Immunol Methods 1983;64:313320.

18. Matta H, Gopalakrishnan R, Choi S, et al.: Development and characterization of a novel luciferase based cytotoxicity assay. Sci Rep 2018;8:199.

19. Schafer H, Schafer A, Kiderlen AF, Masihi KN, Burger R: A highly sensitive cytotoxicity assay based on the release of reporter enzymes, from stably transfected cell lines. J Immunol Methods 1997;204:89-98.

20. Rabinovich PM, Zhang J, Kerr SR, et al.: A versatile flow-based assay for immunocyte-mediated cytotoxicity. J Immunol Methods 2019;474:112668.

21. Sheehy ME, McDermott AB, Furlan SN, Klenerman $P$, Nixon DF: A novel technique for the fluorometric assessment of $\mathrm{T}$ lymphocyte antigen specific lysis. J Immunol Methods 2001;249:99-110.

22. Vermes I, Haanen C, Steffens-Nakken H, Reutelingsperger C: A novel assay for apoptosis. Flow cytometric detection of phosphatidylserine expression on early apoptotic cells using fluorescein labelled Annexin V. J Immunol Methods 1995;184:39-51.

23. Cerignoli F, Abassi YA, Lamarche BJ, et al.: In vitro immunotherapy potency assays using real-time cell analysis. PLoS One 2018;13:e0193498.

24. Mason RD, Welles HC, Adams C, et al.: Targeted isolation of antibodies directed against major sites of SIV Env vulnerability. PLoS Pathog 2016;12:e1005537.

25. Hanawa $\mathrm{H}$, Hematti $\mathrm{P}$, Keyvanfar $\mathrm{K}$, et al.: Efficient gene transfer into rhesus repopulating hematopoietic stem cells using a simian immunodeficiency virus-based lentiviral vector system. Blood 2004;103:4062-4069.

26. Trobridge GD, $\mathrm{Wu} \mathrm{RA}$, Hansen $\mathrm{M}$, et al.: Cocalpseudotyped lentiviral vectors resist inactivation by human serum and efficiently transduce primate hematopoietic repopulating cells. Mol Ther 2010;18:725-733. 
27. Wei X, Decker JM, Liu H, et al.: Emergence of resistant human immunodeficiency virus type 1 in patients receiving fusion inhibitor (T-20) monotherapy. Antimicrob Agents Chemother 2002;46:1896-1905.

28. Turtle CJ, Hanafi LA, Berger C, et al.: CD19 CAR-T cells of defined CD4+:CD8+ composition in adult B cell ALL patients. J Clin Invest 2016;126:2123-2138.

29. Walker RE, Bechtel CM, Natarajan V, et al.: Long-term in vivo survival of receptor-modified syngeneic $T$ cells in patients with human immunodeficiency virus infection. Blood 2000;96:467-474.

30. Sommermeyer D, Hudecek M, Kosasih PL, et al.: Chimeric antigen receptor-modified $\mathrm{T}$ cells derived from defined CD8+ and CD4+ subsets confer superior antitumor reactivity in vivo. Leukemia 2016;30:492-500.

31. Schindler M, Münch J, Brenner M, Stahl-Hennig C, Skowronski J, Kirchhoff F: Comprehensive analysis of nef functions selected in simian immunodeficiency virusinfected macaques. J Virol 2004;78:10588-10597.

32. McBrien JB, Kumar NA, Silvestri G: Mechanisms of CD8(+) $\mathrm{T}$ cell-mediated suppression of HIV/SIV replication. Eur J Immunol 2018;48:898-914.

33. Mahajan SD, Aalinkeel R, Schwartz SA, Chawda RP, Nair MP: Effector cell mediated cytotoxicity measured by intracellular Granzyme B release in HIV infected subjects. Biol Proced Online 2003;5:182-188.
34. Hersperger AR, Makedonas G, Betts MR: Flow cytometric detection of perforin upregulation in human CD8 T cells. Cytometry A 2008;73:1050-1057.

35. Packard BZ, Telford WG, Komoriya A, Henkart PA: Granzyme B activity in target cells detects attack by cytotoxic lymphocytes. J Immunol 2007;179:3812-3820.

36. Yang H, Yorke E, Hancock $\mathrm{G}$, et al:: Improved quantification of HIV-1-infected CD4+ T cells using an optimised method of intracellular HIV-1 gag p24 antigen detection. J Immunol Methods 2013;391:174-178.

37. Perez EE, Wang J, Miller JC, et al.: Establishment of HIV1 resistance in CD4+ T cells by genome editing using zincfinger nucleases. Nat Biotechnol 2008;26:808-816.

38. Egelhofer M, Brandenburg G, Martinius H, et al.: Inhibition of human immunodeficiency virus type 1 entry in cells expressing gp41-derived peptides. J Virol 2004;78:568-575.

Address correspondence to:

Lawrence Corey

Vaccine and Infectious Disease Division Fred Hutchinson Cancer Research Center 1100 Fairview Avenue North, E3-300 Seattle, WA 98109

USA

E-mail:1corey@fredhutch.org 